\title{
Recanalization of Acute Left Internal Mammary Artery Graft Failure by Percutaneous Coronary Intervention
}

\section{Moustapha Diakite ${ }^{1}$, Murat Cimci ${ }^{1}$, Mustafa Cikirikcioglu², Marc-Joseph Licker ${ }^{3}$, Christopher Huber ${ }^{2}$ and Marco Roffi ${ }^{*}$}

${ }^{1}$ Interventional Cardiology Unit, Division of Cardiology, Geneva University Hospitals, Geneva, Switzerland

${ }^{2}$ Division of Cardiovascular Surgery, Geneva University Hospitals, Geneva, Switzerland

${ }^{3}$ Department of Anesthesiology, Geneva University Hospitals, Geneva, Switzerland

*Corresponding author: Marco Roffi, Director, Interventional Cardiology Unit, Division of Cardiology, Geneva University Hospitals, Geneva, Switzerland, Tel: +41 7955 33391; +41 223723 743; Fax: +41 223727 229; E-mail: marco.roffi@hcuge.ch

Rec Date: July 31, 2017, Acc Date: August 12, 2017, Pub Date: August 14, 2017

Citation: Diakite M, Cimci M, Cikirikcioglu M, Licker MJ (2017) Recanalization of Acute Left Internal Mammary Artery Graft Failure by Percutaneous Coronary Intervention. Med Case Rep. Vol.3, No.4: 33.

\section{Abstract}

Acute occlusion of the left internal mammary artery (LIMA) immediately following coronary artery bypass surgery (CABG) is a rare complication that is usually treated by repeat emergency surgery. We described the case of a 65-year-old man known for dyslipidemia and family history of coronary artery disease who was admitted for non-ST elevation myocardial infarction as a result of a severe stenosis of the left anterior descending (LAD) coronary artery. After the discussion within the Heart Team, the patient underwent CABG with LIMA to LAD. As intraoperative blood flow control by transit time flowmeter showed intermittent flow despite optimal anastomosis, immediate coronary angiogram was performed following CABG and an occlusion of the LIMA graft was documented. By contrast injection though a microcatheter advanced into the occluded LIMA it could be demonstrated that the anastomosis was patent and that flow was obstructed by a focal lesion, most likely due to a bend/torsion of the LIMA or a focal vessel wall hematoma. Following multidisciplinary discussion within the Heart Team, a percutaneous coronary intervention (PCI) with a drug eluting stent of LIMA was performed with a good angiographic result and normalization of the flow. The clinical course of the patient was uneventful. This case suggests that, in selected cases, emergent $\mathrm{PCl}$ may be an alternative to repeat-CABG in patients with acute perioperative LIMA occlusion.

Keywords: Transthoracic echocardiography; Dyslipidemia; Coronary angiogram; Negative T-waves

Abbreviations: LIMA: Left Internal Mammary Artery; CABG: Coronary Artery Bypass Grafting; LAD: Left Anterior Descending; $\mathrm{PCl}$ : Percutaneous Coronary Intervention

\section{Case Presentation}

A 65-year-old male, with dyslipidemia and family history of coronary artery disease as cardiovascular risk factors, was admitted to the emergency department for a first episode of typical chest pain lasting for 20 minutes. Physical examination was unremarkable. The electrocardiogram showed negative Twaves in the anterior leads. Transthoracic echocardiography depicted a normal global left ventricular function with no wall motion abnormalities. Serum high-sensitivity cardiac troponin$\mathrm{T}$ was mildly elevated at $23 \mathrm{ng} / \mathrm{L}$ (upper limit of normal 14 $\mathrm{ng} / \mathrm{L})$. The patient was diagnosed with non-ST elevation myocardial infarction (NSTEMI) and underwent same day coronary angiography by radial approach.

Coronary angiography showed single-vessel coronary artery disease with a severe ostial LAD stenosis. After the Heart Team discussion, the patient underwent LIMA grafting to LAD under mini-extracorporeal circulation. During surgery, the transit time flowmeter in the LIMA graft showed intermittent flow despite patent anastomosis. For that reason, emergent coronary angiography was performed immediately after surgery, which showed an occlusion of the LIMA graft (Figure 1). By contrast injection though a microcatheter advanced into the occluded LIMA, it could be demonstrated that the distal portion of the graft as well as the LIMA-LAD anastomosis were patent (Figure 2). Once the microcatheter retracted, flow was restored in the LIMA graft and a focal lesion within the graft was detected, most likely due to a bend/torsion of the LIMA or a focal vessel wall hematoma (Figures 3 and 4). Following Heart Team decision, $\mathrm{PCl}$ of the LIMA lesion was performed and a drug eluting stent (Synergy $3.50 \times 20 \mathrm{~mm}$, Boston Scientific, Marlborough, MA, USA) was placed with good angiographic result while normal flow was restored in the LIMA-LAD territory. In addition to aspirin, the patient received $300 \mathrm{mg}$ of Clopidogrel via the nasogastric tube immediately after stent placement and additional $300 \mathrm{mg}$ six hours later. The clinical course of the patient was uneventful and he was discharged to cardiac rehabilitation on dual antiplatelet therapy, beta-blocker, ACE-inhibitors and high-dose statins. 


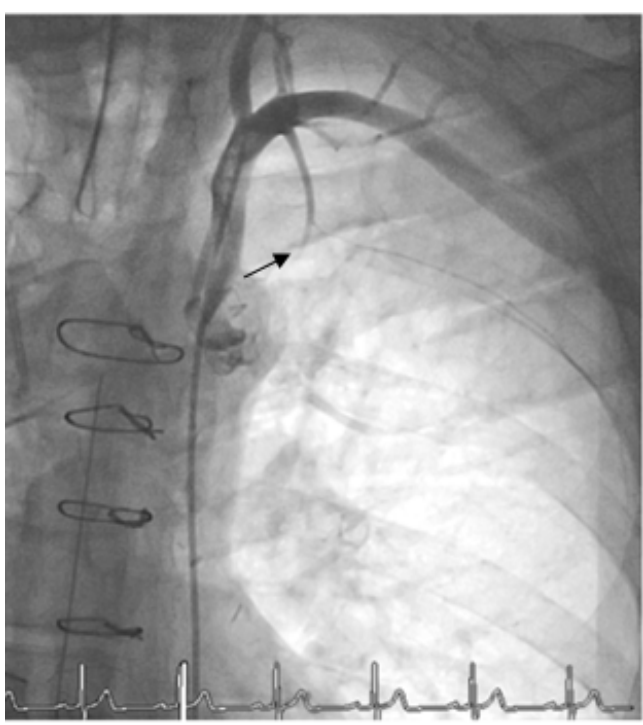

Figure 1 Sub-selective angiogram of the left internal mammary artery showing acute occlusion (arrow).

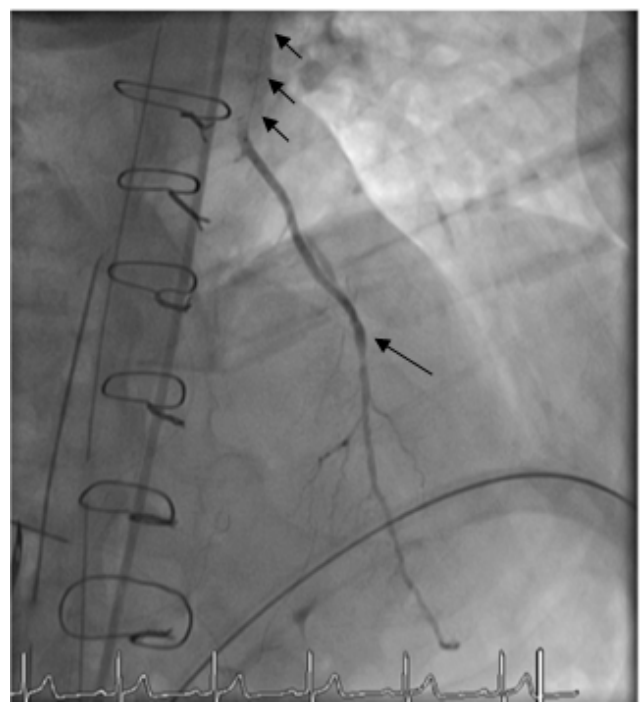

Figure 2 Contrast injections through a microcatheter (small arrows) showing patency of the distal portion of left internal mammary artery graft as well as of the distal anastomosis (long arrow).

\section{Discussion}

Our case illustrates an uncommon complication of CABG, namely an immediate acute LIMA occlusion. While commonly repeat $C A B G$ is considered the treatment of choice in this setting, bailout $\mathrm{PCl}$ was a valuable alternative for our patient.

In CABG it is strongly recommended to use the LIMA as a conduit to the LAD [1]. The benefit of IMA grafts over vein grafts in terms of improved survival is established, independently of left ventricular function, age, sex or stenosis severity [2]. IMA grafts are more resilient than vein grafts to surgical trauma, and endothelial as well as vascular wall functions are better preserved. Moreover, the diameter of IMA grafts and LAD are similar, which limits flow turbulences that may create shear stress-induced endothelial injury. Finally, the endothelial cells of IMA are rich in heparin sulfate and nitric oxide synthase, factors believed to play a crucial role for the superior patency of IMA graft over vein grafts [2].

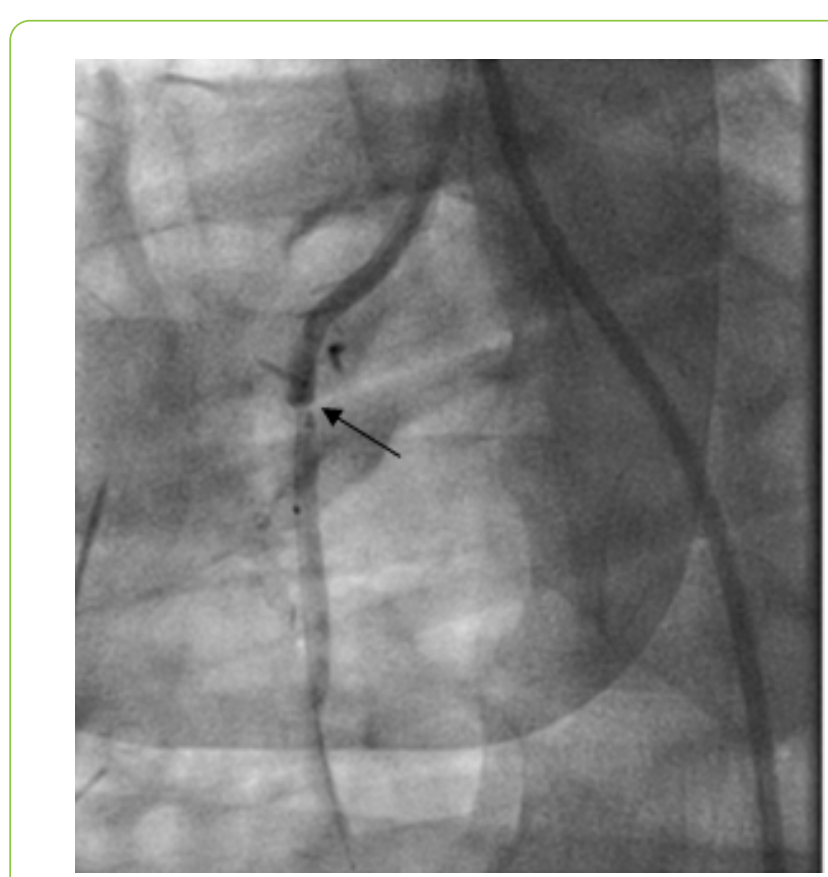

Figure 3 After passing a microcatheter, antegrade flow in the graft is restored and a focal lesion is observed (arrow).

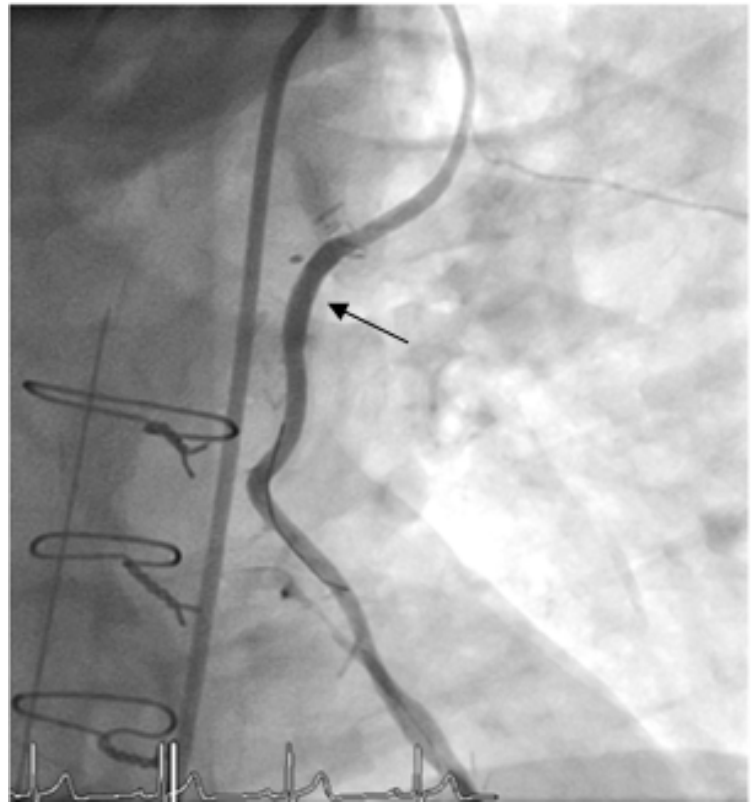

Figure 4 Selective angiogram of the left internal mammary artery graft after stent implantation (arrow).

A study including 366 patients undergoing CABG with the evaluation by intraoperative coronary angiography reported 
up to $12 \%$ early graft failure (LIMA $7 \%$, saphenous vein grafts $8 \%$ ), while around $3 \%$ were clinically evident [3]. Technical issues, especially at the level of the distal anastomosis, are the leading cause of early post-operative IMA failure [2]. Additional causes of early graft failure include conduit defects, poor native vessel run-off, as well as competitive flow with the native vessel [4].

Prevention of post-operative graft failure includes harvesting of the vessels to be used as a conduit with a special attention not to injure the endothelium as well as the assessment of the graft patency and flow at the end of the surgery [5].

As management strategy in case of acute graft failure, the 2014 myocardial revascularization guidelines of the European Society of Cardiology recommend repeat revascularization in case of ongoing myocardial ischemia as well as, in asymptomatic patients, if the artery is of adequate caliber and supplies a large territory; if the indication is established, Heart Team discussion for the treatment options, namely repeat CABG vs. $\mathrm{PCl}$, is led based on the nature of the occlusion, the patient's clinical condition as well as the extent of myocardium at risk [4].

\section{Conclusion}

Although repeat-surgery is commonly considered for acute postoperative LIMA occlusions, our case suggests that, in selected cases, bailout $\mathrm{PCl}$ may be a valid alternative.

\section{References}

1. Hillis LD, Smith PK, Anderson JL, Bittl JA, Bridges CR, et al. (2011) 2011 ACCF/AHA Guideline for Coronary Artery Bypass Graft
Surgery. A report of the American College of Cardiology Foundation/American Heart Association Task Force on Practice Guidelines. Developed in collaboration with the American Association for Thoracic Surgery, Society of Cardiovascular Anesthesiologists, and Society of Thoracic Surgeons. J Am Coll Cardiol 58: e123-210.

2. Harskamp RE, Alexander JH, Ferguson TB Jr, Hager R, Mack MJ, et al. (2016) Frequency and predictors of internal mammary artery graft failure and subsequent clinical outcomes: Insights from the project of ex-vivo vein graft engineering via transfection (PREVENT) IV trial. Circulation 133: 131-138.

3. Zhao DX, Leacche M, Balaguer JM, Boudoulas KD, Damp JA, et al. (2009) Routine intraoperative completion angiography after coronary artery bypass grafting and 1-stop hybrid revascularization results from a fully integrated hybrid catheterization laboratory/operating room. J Am Coll Cardiol 53: 232-241.

4. Windecker S, Kolh P, Alfonso F, Collet JP, Cremer J, et al. (2014) 2014 ESC/EACTS Guidelines on myocardial revascularization The Task Force on Myocardial Revascularization of the European Society of Cardiology (ESC) and the European Association for Cardio-Thoracic Surgery (EACTS) Developed with the special contribution of the European Association of Percutaneous Cardiovascular Interventions (EAPCI). Eur Heart J. 35: 2541-2619.

5. Laflamme M, DeMey N, Bouchard D, Carrier M, Demers $P$, et al. (2012) Management of early postoperative coronary artery bypass graft failure. Interact Cardiovasc Thorac Surg 14: 452-456. 